\title{
CEFALÉIA EPILEPTICA: ESTUDO DE UM CASO COM REGISTRO ELETRENCEFALOGRÁFICO DURANTE A CRISE
}

\author{
ROSI Mary GrossmanN* \\ IRENE ABRaMovich ** \\ ANTONIO B. LEFÈVRE ***
}

\begin{abstract}
Embora sejam freqüentes as referências a cefaléias relacionadas à atividade irritativa cerebral, focal ou difusa, é raro o relato de anormalidades eletrencefalográficas durante crises de cefaléia sem caráter de enxaqueca. $\mathrm{Na}$ literatura a nosso alcance encontramos apenas um caso, descrito por Espadaler-Medina ${ }^{15}$. Por isso julgamos oportuno o relato de um caso referente a uma paciente na qual praticamos o eletrencefalograma (EEG) durante crises de cefaléia, comprovando sua origem epiléptica.
\end{abstract}

\section{O B S E R V A C $\AA$ O}

C.M.S., com 9 anos de idade, sexo feminino, branca (registro H.C. 879.833). A paciente foi internada, em 19-5-69, com queixas de cefaléia em crises e fraqueza muscular generalizada de longa evolução. Na véspera da admissão apresentara, pela primeira vez, três crises convulsivas localizadas no dimidio esquerdo, com generalizaçāo em dua delas. Nos antecedentes da paciente foi averiguado que apresentara retardo do desenvolvimento motor, sỏmente iniciando a marcha aos dois anos de idade e, assim mesmo, apoiada e com grande dificuldade; sòmente aos 7 anos de ldade é que comecou a subir e descer escadas, passando a deambular relativamente bem, porém cansando fácilmente. As crises de cefaléia se iniciaram precocemente, intensificando-se com o cansaço ou ruidos. As cefaléias eram de grande intensidade e ocorriam em crises, geraimente de curta duração. Em duas ocasiōes entretanto, foram de tal modo intensas e duradoras, que motivaram a internação da paciente. O desenvolvimento psiquico da doente sempre foi normal. Não há história de cefaléia semelhante ou convulsões nos familiares. Exame clinico-neurológico - Lordose lombar acentuada, joelhos valgos e pés cavos; ptose palpebral bilateral e hipomimia discretas; déficit muscular axial discreto; marcha e atitude sugestivas de miopatia; reflexos superficiais e profundos normais; sensibilidade normal; fundos oculares normais. Exames complementares - Craniograma normal. Liquido cefalorraqueano normal. Életrencefalograma: intensamente alterado, mostrando anormalidades em ambos os hemisférios cerebrais, mais acentuadas à direita; no hemisfério esquerdo aparece atividade irritativa quase contínua (ondas lentas irregulares, ondas agudas e espiculas), predominando na região occipital; no hemisfério direito foram registradas três crises eletrográficas caracterizadas por ondas agudas ritmadas, atingindo todo o hemisfério, porém iniciadas e predominando em áreas posteriores; essas crises duraram 160, 180 e 200 segundos, respectivamente; nas figuras de 1 a 6 podem ser observados alguns aspectos de uma dessas crises; entre as crises eletro-

Departamento de Neurologia da Faculdade de Medicina da Universidade de São Paulo (Prof. Horácio M. Canelas): * Assistente; ** Residente; *** Professor de Disciplina de Neuropediatria. 
$\mathbf{F 3}-\mathbf{C 3}$

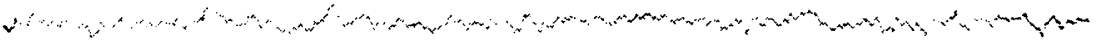

$$
\mathbf{C}-\mathbf{p} \mathbf{3}
$$

CB

$\hat{A}_{\text {F4-C4 }}^{B-01}$

F4-C

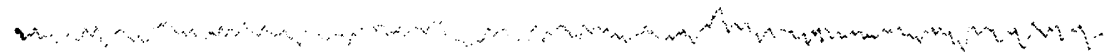

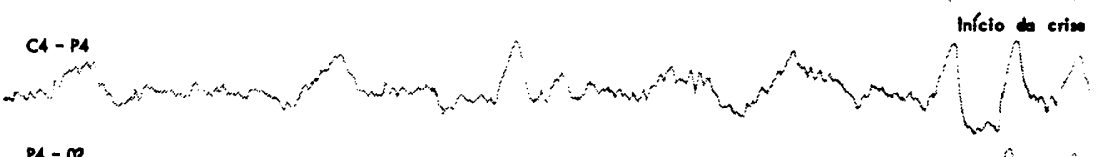

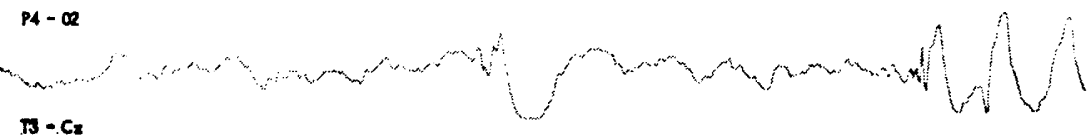

4ha $T 4-C_{2}$

Mryon

Fig. 1 - Infcio da crise eletrográfica, na região parieto-occipital direita, caracterizado por ondas agudas intercaladas a ondas lentas de voltagem elevada.

Po $-C_{3}$

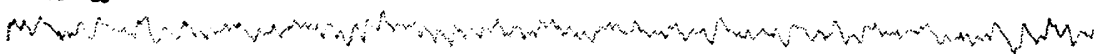

$\boldsymbol{c o s}$

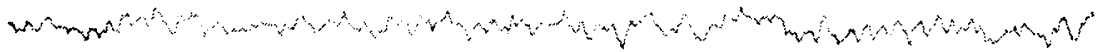

P3 - 01

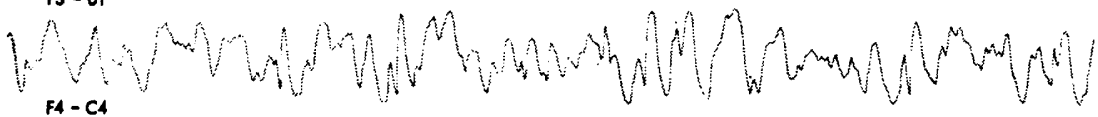
An

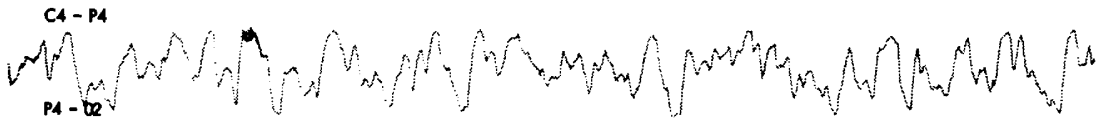

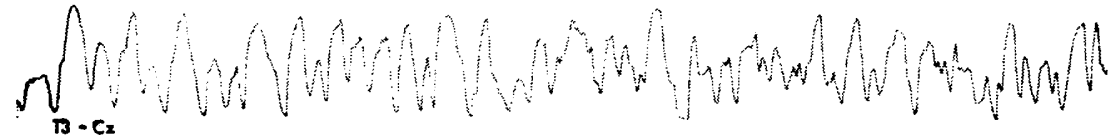

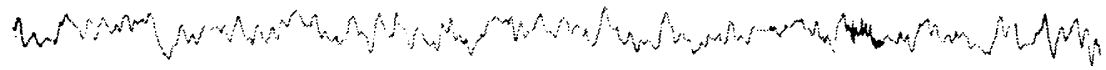
T4 - Cx

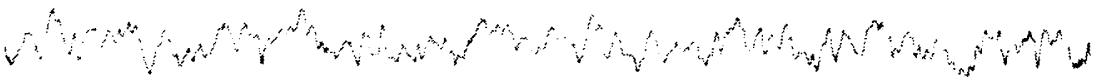

F'ig. 2-Continuação da anterior, nota-se que as ondas lentas ficam progressivamente mais ripida $e$ intercaladas a elementos irritativos. 


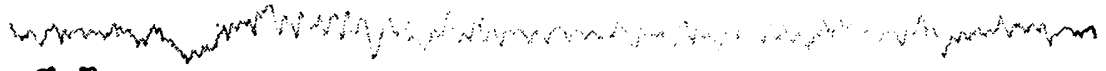
a-B

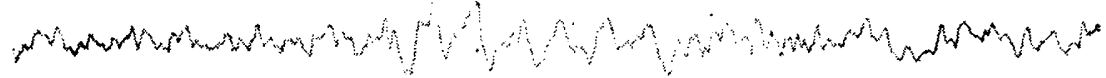

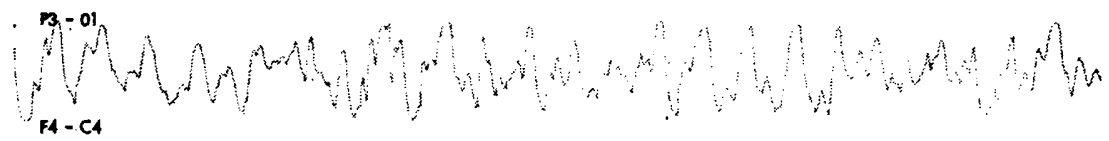

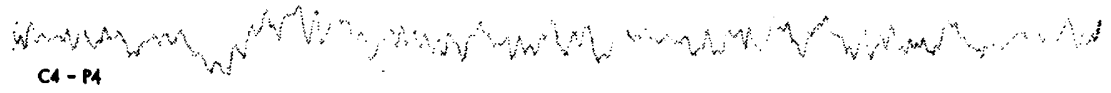

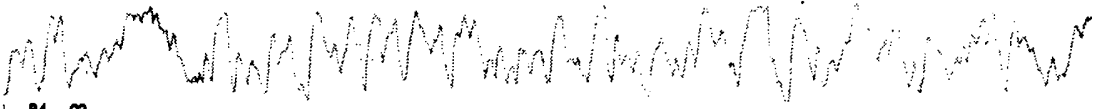
P4 -

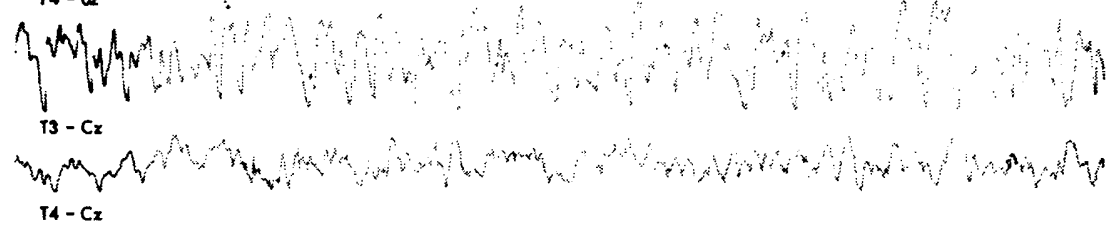

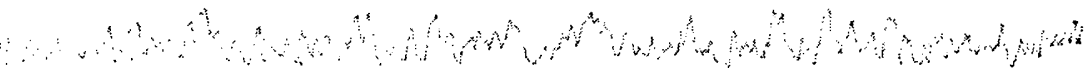

Fig. s - Continuação da anterior, mostra que as ondas na região párietooccipital direita ficaram progressivamente mais rápidas e com maior número de elementos irritativos intercalados.

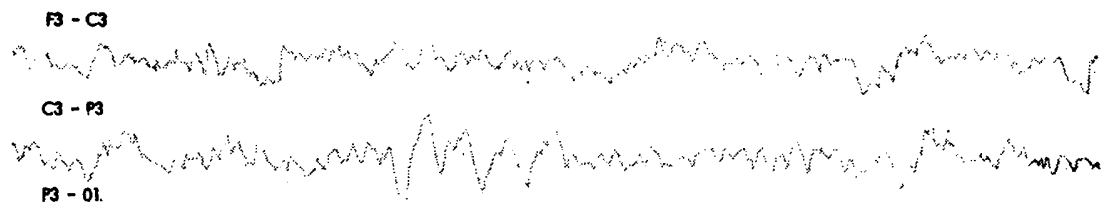

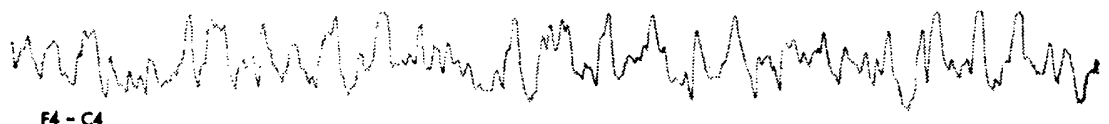
at-P4 "1

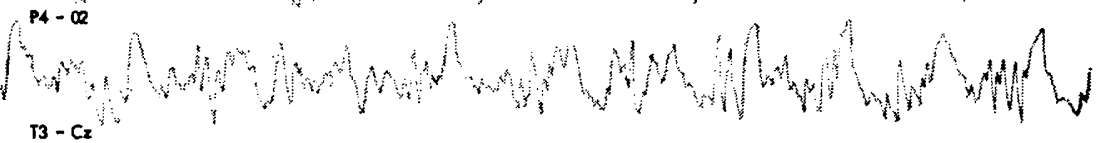

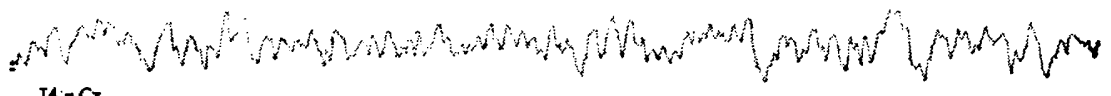
$\mathrm{TH}-\mathrm{Cx}$

aी

Fig. 4 - Cinqüenta segundos após o traçado representado na figura anterior, verifica-se menor número de ondas lentas e predominio de elementos irritativos, agora mais difusos. 


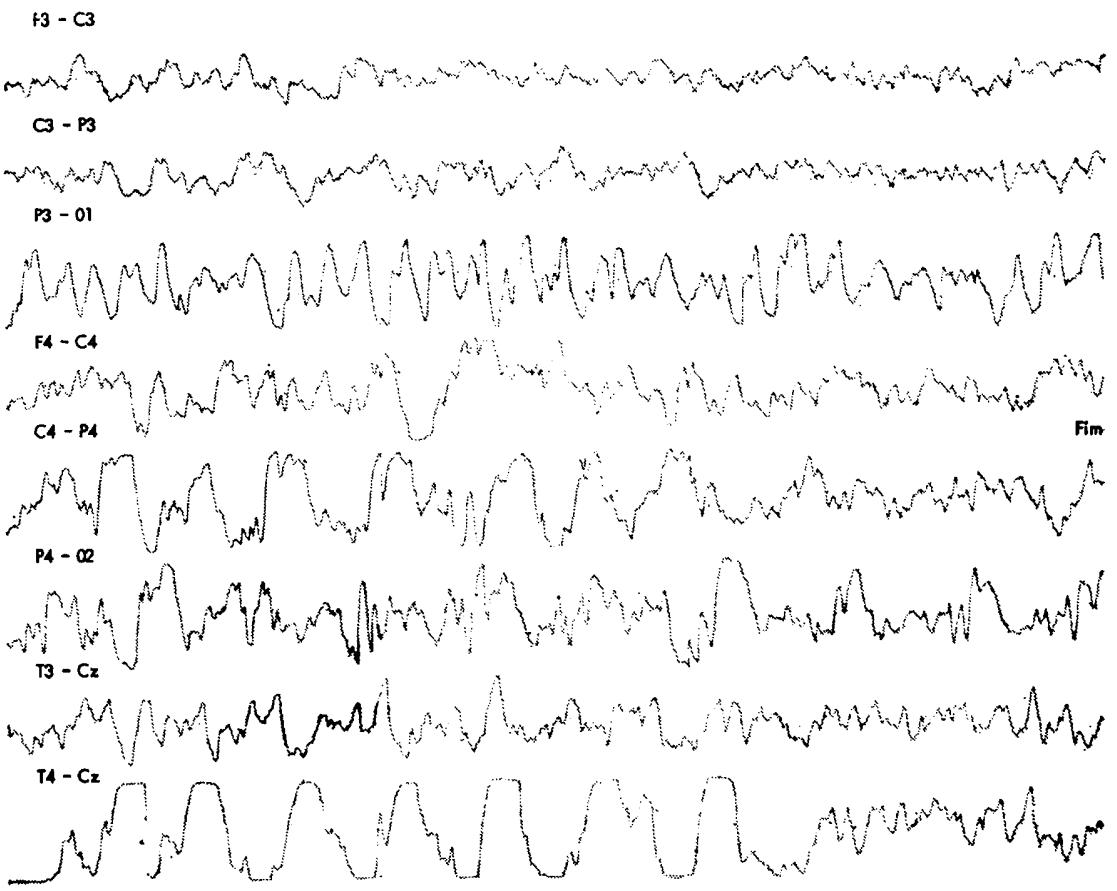

Fig. 5 - Noventa segundos após o tracado representado na figura anterior, observa-se predominio de ondas lentas de voltagem elevada com elementos irritativos mais raros, e o fim da crise.

$B-C$

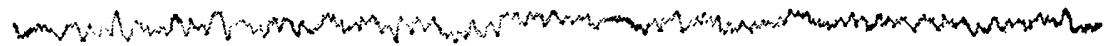

Ca $-\mathrm{P3}$

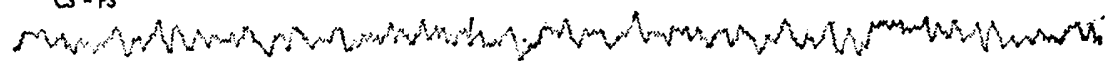

"M Fa-CA Pó arbo

C4-PA

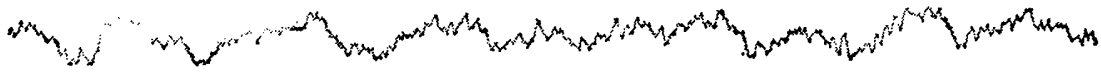

$P 4-\infty$

${ }_{T 3-C_{2}}$

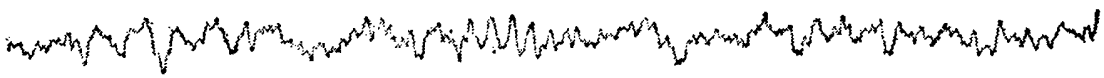
T4-Cz

Hing

Fig. 6 - Continuacão da anterior, notam-se ondas lentas de 1 a $1,5 \mathrm{~Hz}$ de baixa voltagem, predominando na regiäo parieto-occipital direita, indicando sofrimento cerebral pos-crise. 
$\mathrm{F} 3-\mathrm{C} 3$

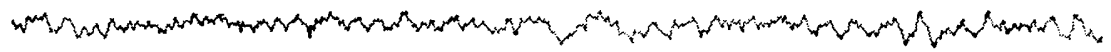
C $-P 3$

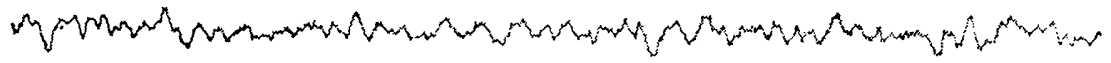

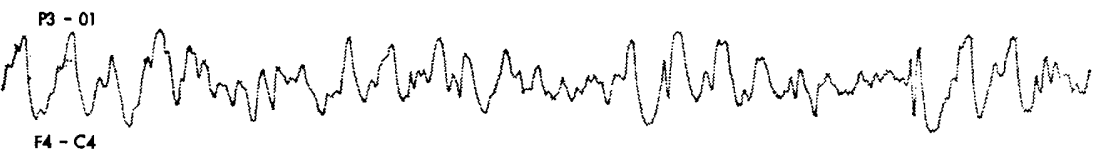

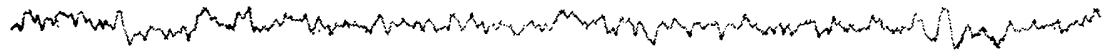
ci-pu

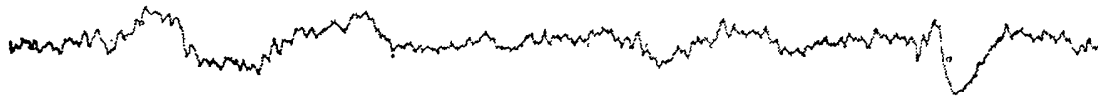
P4.-02

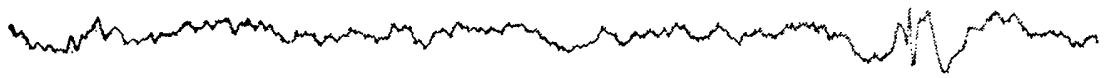
$\mathrm{T}_{3}-\mathrm{CX}_{2}$

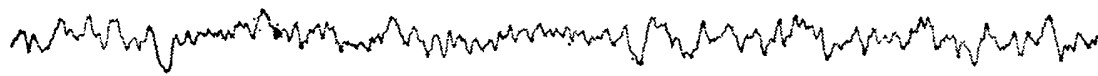
$14-C_{2}$

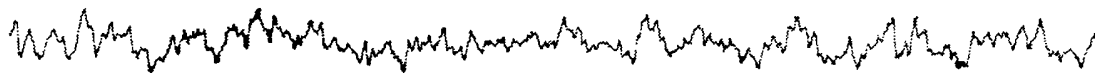

Fig. 7 - Aspecto do EEG observado no período intercrítico. No hemisfério direito, principalmente na regiāo parieto-occipital, notam-se ondas lentas de 1,5 a $2 \mathrm{~Hz}$ de baixa voltagem $e$ uma onda aguda de projeção predominante na região parieto-occipital.

F3- C3

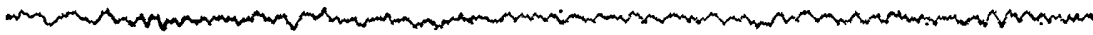
C3-T3.

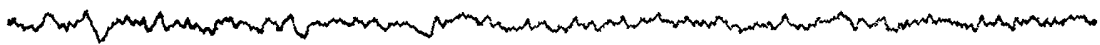
T3 - P3

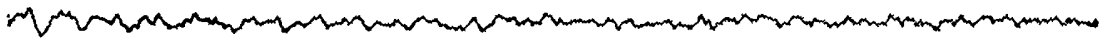
$P 3-01$

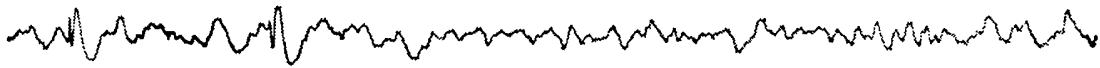
F4 - C4

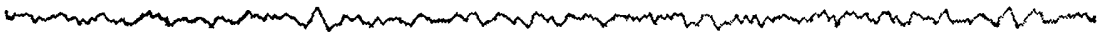
C4 - T4

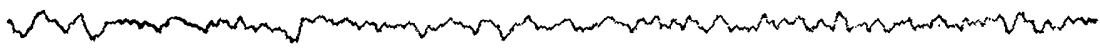
T4-P4

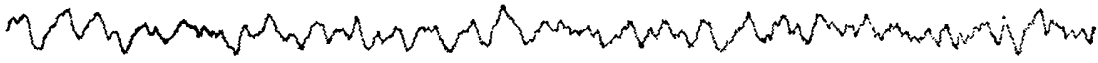
P4 -02

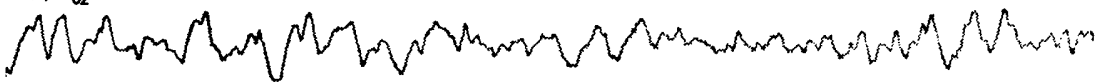

Fig. 8 - EEG feito $s$ meses e 10 dias apos o uso de medicacão anti-convulsivante. Nota-se atividade elétrica cerebral difusamente lenta e pouco organizada, principalmente nas regiōes occipitais onde aparecem ondas de $8 \mathrm{~Hz}$, mescladas a ondas de $\$$ a $4 \mathrm{~Hz}$, aparecendo, além disso, surtos raros de ondas agudas na região occipital esquerda. 
gráficas observou-se sofrimento cerebral (ondas lentas de 1 a $2 \mathrm{~Hz}$ com reduzida amplitude), além de surtos esporádicos de ondas agudas predominantemente parietooccipitais (Fig. 7); coincidindo com essas crises eletrográficas a paciente queixou-se de cefaléia intensa, sem outra sintomatologia. Pan-angiografia (via artéria carótida direita) normal. Pneumoventriculografia normal.

Foi feito o diagnóstico clínico de miopatia de causa não esclarecida associada a epilepsia, sendo esta última traduzida pelas crises de cefaléia e convulsōes. Medicada com Hidantal (2 comp./dia), Ortenal (1,5 comp./dia) e Tegretol (3 comp./dia) a paciente melhorou acentuadamente, nāo apresentando mais crises convulsivas nem cefaléia durante os 4 meses em que a mantivemos sob observação. Repetido o EEG, em 28-8-69, ainda foi evidenciada atividade lenta e pouco organizada, principalmente nas regiões posteriores, onde havia ondas de $8 \mathrm{~Hz}$, muito mescladas a ondas de 3 a $4 \mathrm{~Hz}$, aparecendo, além disso, surtos raros de ondas agudas na regiāo occipital esquerda (Fig. 8). No entanto, a melhora clínica nos autoriza a afirmar ter sido a medicação antiepiléptica eficiente no contrôle das crises de cefaléia e das convulsões.

\section{COM E N T A R I O S}

Há muito tempo vem sendo suspeitada a possível relação entre a enxaqueca e a epilepsia, em virtude da semelhança de algumas características clínicas dessas duas entidades. Com o advento da eletrencefalografia iniciou-se a pesquisa de anormalidades da atividade elétrica cerebral relacionadas às cefaléias crônicas. Engel, Ferris e Romano ${ }^{13}$, foram os primeiros a demonstrar alterações eletrencefalográficas durante crises de enxaqueca. A seguir, Dow e Whitty ${ }^{10}$, descreveram anormalidades do EEG no período intercrítico. Em 1952 Weill $^{42}$ postulou o conceito da enxaqueca disrítmica, agrupando os casos de exaqueca, por vêzes acompanhada de afasia ou quadro sincopal, com alterações eletrencefalográficas, e que respondiam bem à terapêutica anticonvulsivante, sendo rebeldes ao tratamento convencional. Desde então, vários trabalhos têm sido publicados, alguns com o objetivo de provar a relação entre enxaqueca e epilepsia ${ }^{2,8,23,25,29}$ e outros com a intenção de negá-la 1, 3, $4,5,7,9,16,24,39$. As cefaléias crônicas paroxísticas, não enquadráveis dentro do conceito da enxaqueca, também têm sido estudadas sob tal aspecto ${ }^{18,20,21}$, $27,28,30,33,40,43$. Observa-se na literatura, entretanto, que a maioria dos trabalhos relata anormalidades eletrencefalográficas no periodo intercrítico da enxaqueca ou, mais raramente, de outros tipos de cefaléia. Os achados eletrencefalográficos durante crises de cefaléia têm sido relatados apenas em casos de enxaqueca. Durante essas crises o EEG costuma ser normal 6, 10, 19, 24 . Segundo Kunkle ${ }^{24}$, em duas eventualidades, porém, o EEG pode ser patológico: 1) se fôr feito durante os pródromos da enxaqueca, sendo êstes visuais, pode ser encontrada, na região occipital correspondente, maior quantidade de ritmos lentos; 2) se a enxaqueca fôr acompanhada por déficit neurológico, como hemiparesia ou disfasia, é provável que, durante a crise ou logo após a mesma, apareçam anormalidades no EEG. Nessas circunstâncias o EEG mostra ondas delta em um dos hemisférios cerebrais, ou difusas, predominando em um dos lados, indicando sofrimento cerebral 7, 10, 11, 12, 13, 14, 19, 22, 26, 31, 32, 34, 36, 37, 38, 41. Essas ondas lentas são resultantes do vasospasmo existente na enxaqueca, descrito por Schumacher e Wolff ${ }^{35}$. Tais 
anormalidades eletrencefalográficas persistem durante horas ou dias, desaparecendo geralmente com os sinais clínicos.

Sòmente encontramos o registro de um caso publicado por EspadalerMedina ${ }^{15}$, que menciona os achados eletrencefalográficos durante crise de cefaléia com início e fim abruptos e duração de 8 segundos; concomitantemente apareceram, no traçado, complexos de espiculo-onda, bilaterais e síncronos, com $2,5 \mathrm{~Hz}$.

O caso ora relatado indica que crises de cefaléia, sem caráter de enxaqueca, podem ser de natureza epiléptica, pois as três crises referidas durante o registro eletrencefalográfico foram simultâneas com o aparecimento, no traçado, de anormalidades de tipo irritativo, ou seja, crises eletrográficas caracterizadas por ondas agudas ritmadas.

Alguns autores 1, 17, 39 não aceitam a classificação da enxaqueca dentro do conceito de epilepsia, argumentando que, embora na enxaqueca existam anormalidades de tipo irritativo no periodo intercrítico, durante as crises, quando existem anormalidades eletrencefalográficas, estas não são do tipo irritativo, mas sim indicativas de sofrimento cerebral.

Nosso caso, bem como o publicado por Espadaler-Medina ${ }^{15}$, mostra que algumas cefaléias crônicas, que evoluem em crises, geralmente de inicio e fim abruptos e de curta duração, sem causa orgânica aparente, podem ser perfeitamente clasificadas dentro do conceito de epilepsia, pois, além de apresentarem anormalidades irritativas no período intercrítico, também as exibem na vigências das crises de cefaléia. Tais cefaléias, assim como as enxaquecas disritmicas, evoluem favoràvelmente com o emprêgo de anticonvulsivantes. Deve-se salientar que nestes casos, associados às crises de cefaléia, podem ser encontrados outros sintomas epilépticos, tais como crises convulsivas, fato verificado nos pacientes observados por nós e por EspadalerMedina ${ }^{15}$.

\section{R E S U M O}

É relatado o caso de uma paciente com 9 anos de idade, na qual foi feito - EEG durante 3 crises de cefaléia sem caráter de enxaqueca. Simultâneamente com as crises foram observadas, no EEG, anormalidades caracterizadas por ondas agudas ritmadas de projeção difusa no hemisfério cerebral direito, predominando na região occipital. São feitos comentários sôbre a raridade do relato de anormalidades do tipo irritativo durante crises de cefaléia, sendo ressaltado o valor de tais achados para a classificação dessas cefaléias dentro do conceito de epilepsia.

\section{S U M M A R Y}

Epileptic headache: report of a case with electroencephalogram recorded during the crisis

The case of a 9-year-old patient, in which the EEG was made during three crises of headache without the character of migraine is reported. Simultaneously with the crises the EEG showed rhythmic sharp waves in 
the right cerebral hemisphere prevailing in the occipital region. Comments are made on the rarity of reports showing irritative abnormalities in the EEG during headache crises. The value of these findings for the classification of some cases of headache within the concept of epilepsy is emphasized.

\section{RE F E R E N I A S}

1. AKIмото, H. - Electroencephalographic studies in migraine. Int. J. Neurol. (Montevideo) 3:379, 1962.

2. ARIAS, B. R.; DIES, J. M. S. \& BADO, J. V. - Estudio EEG de una serie de migrañosos. Electroenceph. clin. Neurophysiol. 5:461, 1953.

3. BAROLIN, G. S. - The EEG in atypical migraine. Electroenceph. clin. Neurophysiol. 17:348, 1964.

4. BAROLIN, G. S. - Migraines and epilepsies - a relationship? Epilepsia (Amsterdam) 7:53, 1966.

5. BAROLIN, G. S. - The relationship between migraine and epilepsy. Electroenceph. clin. Neurophysiol. 20:106, 1966.

6. BOUDIN, G.; PEPIN, B.; BARBIZET, J. \& MASSON, S. - Migraine and EEG disturbances. Electroenceph. clin. Neurophysiol. 14:141, 1962.

7. CAMP, W. A. \& WOLFF, H. G. - Studies on headache. Arch. Neurol. 4:475, 1961.

8. ChaVANY, J. A.; LOBEL, G. \& HAGENMUlleR, D. - L'epilepsie pseudo-migraineuse. Sem. Hôp. Paris 30:1626, 1954.

9. COTTE-RITTAUD, M. R. - EEG abnormalities observed in patients with migraine. Electroenceph. clin. Neurophysiol. 17:720, 1964.

10. DOW, D. J. \& WHITTY, C. W. M. - Electro-encephalographic changes in migraine. Lancet 253 ii:52, 1947.

11. EDES, A. O. \& DALY, D. D. - The EEG in a particular variety of migraine. Electroenceph. clin. Neurophysiol. 19:415, 1965.

12. EDES, A. O. \& DALY, D. D. - EEG studies in complex migraine. Electroenceph. clin. Neurophysiol. 23:86, 1967.

13. ENGEL, G. L.; FERRIS, E. B. \& ROMANO, J. - Focal electroencephalographic changes during the escotomas of migraine. Amer. J. Med. Sci. 209:650, 1945.

14. ENGEL, G. L.; HAMBURGER, W. W.; REISER, M. \& PLUNKETT, J. - Electroencephalographic and psychological studies of a case of migraine with severe preheadache phenomena. Psychosom. Med. 15:337, 1953.

15. ESPADAler-MEDINA, J. M. - Céphalées epileptiques. World Neurol. 2:309, 1961.

16. FELDMAN, R. G. \& GLASER, G. H. - EEG study of methysergide in migraine. Electroenceph. clin. Neurophysiol. 15:699, 1963.

17. FRIEDMAN, A. P. \& MERRIT, H. H. - Migraine. In Friedman, A. P. \& Merrit, H. H.: Headache: Diagnosis and Treatment. F. A. Davis Co. Publishers, Philadelphia, 1959, pp. 201-250.

18. Froelich, W. A.; CARTER, C. C.; O'Leary, J. L. \& ROSEnbaum, H. E. Headache in childhood. Neurology (Minneapolis) 10:639, 1960.

19. GASTAUT, H.; NAVARRANNE, P. \& SIMON y CANTON, L. - EEG characteristics of migrainous cerebral attaks of a deficitary type (hemiplegic migraine). Electroenceph. clin. Neurophysiol. 23:381, 1967.

20. GIEL, R.; VLIEGER, M. \& VLIET, A. G. M. - Headache and the EEG. Electroenceph. clin. Neurophysiol. 21:292, 1966.

21. HALPERN, L. \& BENTAL, E. - Epileptic cephalea. Neurology (Minneapolis) 8:615, 1958.

22. HERON, J. R. - The electroencephalogram in complex forms of migraine. Electroenceph. clin. Neurophysiol. 22:293, 1967.

23. HEYCK, H. - Dolor de Cabeza. Trad. castelhana por C. R. Borstnar. Marin, Barcelona, 1959. 
24. KUNKLE, E. C. - In Merrit, H. H. - Summary and discussion (Symposium on headache: its mechanism, diagnosis and management). Neurology (Minneapolis) 13 (mar. special issue):38, 1963.

25. LENNOX, W. G. - Migraine, an autonomic epilepsy? In Lennox, W. G. \& Lennox, M. A.: Epilepsy and Related Disorders. Little-Brown Boston, 1960, p. 449 .

266. LERIQUE-KOECHLIN, A. \& MISES, J. - L'EEG dans une manifestation paroxystique non-épileptique de l'enfant: la migraine. Electroenceph. clin. Neurophysiol. 16:203, 1964.

27. MATZILEWICH, B. \& MERLIS, J. D. - Headache as an epileptic manifestation. Arch. Neurol. Psychiat. (Chicago) 66:651, 1951.

28. MISES, J. \& LERIQUE, A. - Electroclinical study of chronic headaches in children. Electroenceph. clin. Neurophysiol. 12:941, 1960.

29. MOENCH, L. G. - Migraine. In Moench, L. G.: Headache. The Year Book Publishers Inc., Chicago, 1947, pp. 154-186.

30. OBAAL, F. \& TOMKA, J. - EEG examinations on patients with headaches. Electroenceph. clin. Neurophysiol. 12:935, 1960.

31. O'CONNOR, P. \& DAVIES, R. - Hemiplegic migraine. Electroenceph. clin. Neurophysiol. 19:531, 1965.

32. POLAK, O. \& GROF, P. - An electroencephalographic record in cerebellar migraine and the influence of Ultracorten-H. Electroenceph. clin. Neurophysiol. $20: 283,1966$.

33. PUPO, P. P.; ZUKERMAN, E. \& ACUNA-OLCESTER, E. - Cefaléia: aspectos eletrencefalográficos. Rev. paul. Med. 67:165, 1965.

34. RODIN, E. A. - Paroxysmal atypical headache associated with marked electroencefalographic abnormalities. Electroenceph. clin. Neurophysiol. 12:268, 1960.

35. SCHUMACHER, G. A. \& WOLFF, H. G. - Experimental studies on headache. Arch. Neurol. Psychiat. (Chicago) 45:199, 1941.

36. SMYTH, V. O. G. \& WINTER, A. L. - The EEG in migraine. Electroenceph. clin. Neurophysiol. 13:828, 1961.

37. SMYTH, V. O. G. \& WINTER, A. L. - The EEG in migraine. Electroenceph. clin. Neurophysiol. 16:194, 1964.

38. TARLAU, M.; ALlAN, D. F. \& POMINA, A. - Dysrhythmic migraine with unusual clinical and EEG features. Electroenceph. clin. Neurophysiol. 13:496, 1961.

39. TASHEV, T. - Electroencephalographic findings in migraine and chronic headache. Electroenceph. clin. Neurophysiol. 15:716, 1963.

40. ULETT, G. A.; EVANS, D. \& O'LEARY, J. L. - Survey of EEG, findings in 1.000 patients with chief complaint of headache. Electroenceph. clin. Neurophysiol. $4: 463,1952$.

41. UZUNOV, N. - Some clinic-electrographic correlations in migrainous children. Electroenceph. clin. Neurophysiol. 17:709, 1964.

42. WEILL, A. A. - EEG findings in a certain type of psychosomatic headache: dyshythmic migraine. Electroenceph. clin. Neurophysiol. 4:181, 1952.

43. ZIEGLER, D. K. \& TORRES, F. - Focal spike and wave activity in a patient with headaches. Electroenceph. clin. Neurophysiol. 8:133, 1956.

Clinica Neurológica - Faculdade de Medicina, Universidade de São Paulo Caixa Postal 3461 - São Paulo, SP - Brasil. 